\title{
ALIGNED ELECTROSPUN POLYMER FIBRES FOR SKELETAL MUSCLE REGENERATION
}

\author{
K.J. Aviss, J.E. Gough, and S. Downes* \\ School of Materials, Biomaterials Group, The University of Manchester, Manchester, M1 7HS, UK
}

\begin{abstract}
Skeletal muscle repair is often overlooked in surgical procedures and in serious burn victims. Creating a tissueengineered skeletal muscle would not only provide a grafting material for these clinical situations, but could also be used as a valuable true-to-life research tool into diseases affecting muscle tissue. Electrospinning of the elastomer PLGA produced aligned fibres that had the correct topology to provide contact guidance for myoblast elongation and alignment. In addition, the electrospun scaffold required no surface modifications or incorporation of biologic material for adhesion, elongation, and differentiation of C2C12 murine myoblasts.
\end{abstract}

Keywords: Electrospinning, myoblasts, alignment, PLGA, surface topography.

*Address for correspondence:

Sandra Downes

School of Materials, Biomaterials Group

The University of Manchester

Manchester, M1 7HS, UK

E-mail: sandra.downes@manchester.ac.uk

\section{Introduction}

Skeletal muscle is a highly organised and hierarchical tissue. Creating and maintaining myoblast to myotube morphology has been a challenge in tissue engineering of skeletal muscle (Huang et al., 2006; Riboldi et al., 2005). Grown on surfaces with no contact guidance, myoblasts grow in random swirling patterns; which is not conducive to the formation of efficient contraction. In order to contract as a syncytium, muscle fibres must grow parallel to one another with identical anisotropy. This can be done by using a scaffold with the relevant topography to induce this behaviour via contact guidance (Bashur et al., 2006; Dalby et al., 2003). It is known that the structural components of the extracellular matrix (ECM) and their interaction with transmembrane proteins, such as integrins play a role in the organisation of tissues, including the organisation of myoblasts (Bray et al., 2008; Schiaffino and Partridge, 2008). These ECM cues provide topology and mechanical properties relevant to the tissue and changes in ECM structure can affect cellular behaviour. To aid the regeneration of skeletal muscle, an oriented scaffold that provides a template for alignment could be used to encourage this organisation in myoblasts as they fuse and differentiate to form multinucleated myofibres. By electrospinning an elastomeric polymer, poly(lactideco-glycolide), it is possible to create such a scaffold that may provide the topographical cues and contact guidance for alignment of myoblasts (Choi et al., 2008), while providing an elastic substrate for myotube differentiation. The technique of electrospinning is attractive to use in this application because of the amount of control available by altering various parameters. For example, the size of the fibres, from the micrometer to the nanometre range, can be controlled by changing the concentration of polymer, flow rate and the distance from needle to collector plate. A comprehensive review of the electrospinning process has been carried out by Subbiah et al. (2004).

Alignment of polymer nanofibres can be controlled by selecting the relevant collector plate: stationary or very slow rotation of the collector plate can be used to manufacture randomly oriented fibres, whereas a highspeed rotating mandrel can be used to create aligned fibres (Bian and Bursac, 2009; Blackwood et al., 2008; Courtney et al., 2006; Wang et al., 2009).

To date polyesters such as PCL (polycaprolactone), PLA (poly (lactic acid)), PGA (poly (glycolic acid)), and their co-polymer PLGA (poly (lactide-co-glycolide)) have been used in the form of foams, films, and electrospun fibres for tissue engineering applications (Huang et al., 2006; Huang et al., 2005; Singh et al., 2004). Levenberg et al. (2005) used a sponge of PLLA (poly-L-lactic acid) and PLGA to form a highly porous three-dimensional (3D) 
scaffold for vascularised skeletal muscle development, with the addition of Matrigel for increased cell infiltration. Choi et al. (2008) electrospun PCL with collagen to form a biodegradable, oriented, fibrous scaffold to induce myotube alignment. Both these studies used polyesters that incorporated biologic materials to enhance cell attachment; these biologic components decrease relevancy for clinical applications. Inclusion of animal products, e.g., collagen and Matrigel could provoke an immune response in a host if used as an implantable construct. Eliminating these biologic materials by using a totally synthetic and controllable polymer decreases the risk of an immune response to the construct. A totally synthetic biocompatible scaffold for skeletal muscle engineering, which includes topographical cues for myoblast alignment, would therefore be advantageous.

Previous work in skeletal muscle regeneration has shown that using flat, inflexible scaffolds for myoblast differentiation is ineffective as the myoblasts spontaneously contract as they near the end of the differentiation process and often detach away from stiff scaffolds (Cooper et al., 2004; Engler et al., 2004; Ren et al., 2008). Using the elastomeric polymer PLGA may overcome this problem thus creating a scaffold which may be compatible for long term culture of skeletal muscle cells, with potential for creation of a 3D tissue construct.

PLGA undergoes elastic deformation in response to cyclic strain (Webb et al., 2004), as is expected from an elastomeric polymer. For this study the scaffold needs to withstand the stresses and strains of a monolayer of differentiating myoblasts into myotubes. An elastic, yet comparatively stiff scaffold would allow myoblasts to differentiate into myotubes without them detaching from the surface (Engler et al., 2004). Levy-Mishali et al. (2009) investigated a range of PLGA scaffolds with differing stiffnesses and found that those with a Young's modulus of greater than $200 \mathrm{kPa}$, such as their PL75GA25 enabled myotube formation, and that those with a lower Young's modulus value collapsed under the forces of the myotubes (Levi-Mishali et al., 2009).

Degradation products can be an issue when synthetic polymers are used as biomaterials. PLGA degrades into lactic acid and glycolic acid, both of which occur naturally, and should be easily excreted from the highly metabolic skeletal muscle tissue. It is possible to control the degradation time of PLGA by altering the ratio of lactide:glycolide (Kim et al., 2003; Zong et al., 2003). Groups have researched PLGA degradation and found no change in $\mathrm{pH}$ over 22 days (Zong et al., 2005; Zong et al., 2003). This would indicate PLGA is compatible for longterm tissue culture as a change in $\mathrm{pH}$ could affect cellular growth and survival. The degradation of electrospun PL85GA15 has not been investigated, however, Kaetsu et al. (1987) found solid PL85GA15 degraded in 6 weeks. This may not be the same as for electrospun fibres as bulk phase degradation may not occur due to the small size of the fibres and the ease of water infiltration washing any degradation products away halting autocatalysis.

The objective of this study is to determine whether murine myoblasts $(\mathrm{C} 2 \mathrm{C} 12)$ adhere and proliferate upon an electrospun synthetic polymer (PLGA) scaffold, with no surface modifications to the polymer or the use of a feeder layer; and whether the oriented fibres within the scaffold can provide adequate topographical cues and contact guidance for myoblast elongation and alignment.

\section{Materials and Methods}

\section{Materials}

PDL85GA15 (Average molecular weight $\left(\mathrm{M}_{\mathrm{w}}\right)$ 50,000 70,000), hexafluoroisopropanol (HFIP), and FITCconjugated phalloidin were purchased from Sigma Aldrich (St. Louis, MO, USA). C2C12 murine myoblasts were purchased from the European Collection of Cell Cultures (ECACC) (Sigma Aldrich) (91031101). 4',6-diamidino2-phenylindole (DAPI) Prolong Gold Antifade Reagent was purchased from Invitrogen (Carlsbad, CA, USA). Mouse monoclonal IgG [MY-32] to fast skeletal myosin was purchased from Abcam (Cambridge, MA, USA) (ab7784-250). Sarcomeric myosin was detected using the primary antibody A1025, which was a very generous gift from Dr. Michelle Peckham, University of Leeds. Secondary antibodies were purchased from Invitrogen's Molecular Probes (Alexa Fluor 488 and 546).

\section{Electrospinning}

A $20 \% \mathrm{w} / \mathrm{v}$ solution of PLGA in HFIP was loaded into a $10 \mathrm{ml}$ syringe with a blunted BD (Franklin Lakes, NJ, USA) Microlance $321 \mathrm{G}$ needle. A $1 \mathrm{ml} /$ hour flow rate was applied with a voltage of $25 \mathrm{kV}$, and a needle tip to collector plate distance of $15 \mathrm{~cm}$. A rotating mandrel with dimensions of $11 \mathrm{~cm}$ in length, and $3.5 \mathrm{~cm}$ in diameter, was used as the earthed collector plate. To create randomly oriented fibres the rotation speed was $300 \mathrm{rpm}$, for aligned fibres this was increased to $1500 \mathrm{rpm}$. Annotations to each fibre type will be according to mandrel rotation speed: RPM 300 (random), and RPM 1500 (aligned). Electrospinning produced thin sheets of fibrous meshes, approximately $0.1-0.3 \mathrm{~mm}$ thick. Prior to cell seeding the electrospun sheets were cut to fit into a sterile 12 well plate Scaffdex well insert (C00002S, Scaffdex, Tampere, Finland) to hold them at the bottom of the well plate, and sterilised under UV light in a laminar flow hood for 10 minutes on either side.

\section{Fibre characterisation}

A Topcon (Tokyo, Japan) scanning electron microscope (SEM) was used to visualise the fibrous sheets with and without cells. Cell seeded samples were fixed with $3 \%$ glutaraldehyde for 30 minutes at $4{ }^{\circ} \mathrm{C}$ and rinsed in $0.1 \mathrm{M}$ phosphate buffer. Cellular samples were dehydrated using a series of increasing ethanol concentration incubations followed by an overnight incubation in hexamethyldisilasane (HMDS). Samples were mounted on carbon tabs and gold sputter coated. Fibre diameter and angles for alignment were determined manually from the SEM images using the software ImageJ, and the Students' $t$-test was used to analyse any statistical difference between RPM 300 and RPM 1500 fibres $(n=50$, 10 fibre measurements from 5 different SEM images). Mechanical testing was undertaken using an Instron (High Wycombe, 


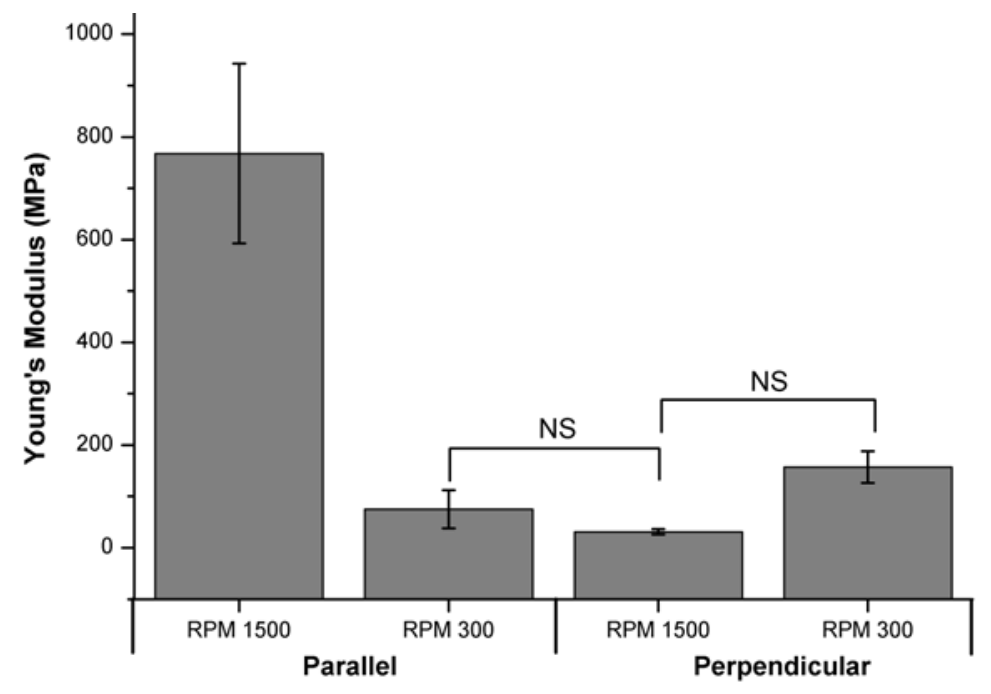

Figure 1: Graph showing Young's modulus of electrospun scaffolds RPM 1500 and RPM 300. Scaffolds were tested along the parallel directional axis of the fibres and perpendicular to the directional axis of the fibres. Bars indicate standard deviation; NS over brackets indicates no significant difference.
U.K.) 1122 tensile tester applying $200 \mathrm{~g} / \mathrm{cm}^{2}$ load at $5 \mathrm{~mm} /$ min until breakage was detected. Breakage was detected when no resistance to load was recorded. Scaffolds were tested along the directional axis of the fibres, i.e., along the length of the fibres as spun $(n=8)$, and perpendicular to the directional axis of the fibres as spun $(n=4)$. Young's modulus was calculated by dividing maximum stress by maximum strain.

\section{Cell culture and seeding}

C2C12 murine myoblasts were cultured in DMEM (Dulbecco's modified Eagle's medium) supplemented with $10 \%$ FBS (foetal bovine serum) and 1\% penicillin/ streptomycin, grown in a humidified incubator at $37^{\circ} \mathrm{C}$, with $5 \% \mathrm{CO}_{2}$. For differentiation induction, the serum content in the growth media was decreased to $5 \%$ after 24 hours. For all studies cells were seeded at 25,000 cells $/ \mathrm{ml}$. Electrospun scaffolds were held at the bottom of a 12 well plate with Scaffdex 12 well plate inserts.

\section{Fluorescence staining}

Cellular morphology and alignment was investigated by fluorescently staining the f-actin cytoskeleton using fluorescein isothiocyanate (FITC)-conjugated phalloidin, and nuclear staining with DAPI-Prolong. Cells were fixed with $3 \%$ paraformaldehyde (PFA) for 15 minutes at room temperature before being permeabilised and blocked using ICC block buffer ( $1 \%$ goat serum, $1 \mathrm{mg} / \mathrm{ml}$ bovine serum albumin (BSA), and $0.1 \%$ Triton $\mathrm{X}-100$ ) at room temperature for 30 minutes. For each sample $5 \mu$ of a 6 $\mu \mathrm{M}$ stock solution of FITC-phalloidin was added to 200 $\mu \mathrm{l}$ ICC buffer and incubated at room temperature for 30 minutes. Samples were analysed using a fluorescence microscope (Nikon Eclipse 50i) (Nikon, Tokyo, Japan) at $10 \mathrm{x}$ and $20 \mathrm{x}$ magnification, and a Leica (Wetzlar, Germany) TCS SP5 confocal microscope for higher magnifications for more detailed inspection of the cytoskeleton. Cell elongation was analyzed by measuring the aspect ratio (length/width) of each cell $(n=20$ per scaffold type) via ImageJ. A Student's $t$-test was used to determine statistical differences between the scaffold types.

Immunostaining was performed to detect adult fast myosin heavy chain differentiation marker. Cells were fixed with 3\% PFA and treated with ICC buffer as above. The primary antibody (mouse monoclonal IgG [MY-32] to fast skeletal myosin - ab7784-250) was added at a 1:1000 dilution in ICC block buffer and incubated at room temperature for 1 hour. Samples were washed twice with PBS and the secondary antibody (goat anti-mouse Alexa Fluor 488) was added at a 1:1000 dilution in ICC block buffer and incubated at room temperature for 1 hour before samples were washed with PBS and mounted with DAPIProlong and visualized under the fluorescence microscope as above. Fusion index was calculated by dividing the number of green positive multi-nucleated myotubes by the total number of nuclei present in the image then multiplying by 100 .

Sarcomeric myosin was detected using the A1025 primary antibody from Dr. Michelle Peckham at a concentration of 1:100 in ICC block buffer and incubated at room temperature for 1 hour. Secondary antibody was goat anti-mouse antibody (Alexa Fluor 546), which was added at 1:1000 in ICC block buffer for 1 hour at room temperature. Sarcomeric banding was visualized using a Leica TCS SP5 confocal microscope.

\section{Results}

\section{Fibre characterisation}

Results in Fig. 1 illustrate the Young's modulus of the electrospun scaffolds. These results show that the RPM 1500 fibres tested along the length of the directional axis as spun are much stiffer, with a higher modulus value than RPM 300 fibres tested in the same way and also when tested with load applied perpendicular to the directional axis.

The differences between the two fibre types tested perpendicular are not significant from each other; there is also no significant difference between RPM 300 fibres tested parallel to the directional axis as spun and the RPM 1500 fibres tested perpendicular. RPM 1500 fibres tested parallel to the directional axis as spun are significantly different to all other fibre types tested either way. Elongation at break was also calculated with RPM 1500 fibres tested parallel to the directional axis as spun, with a 

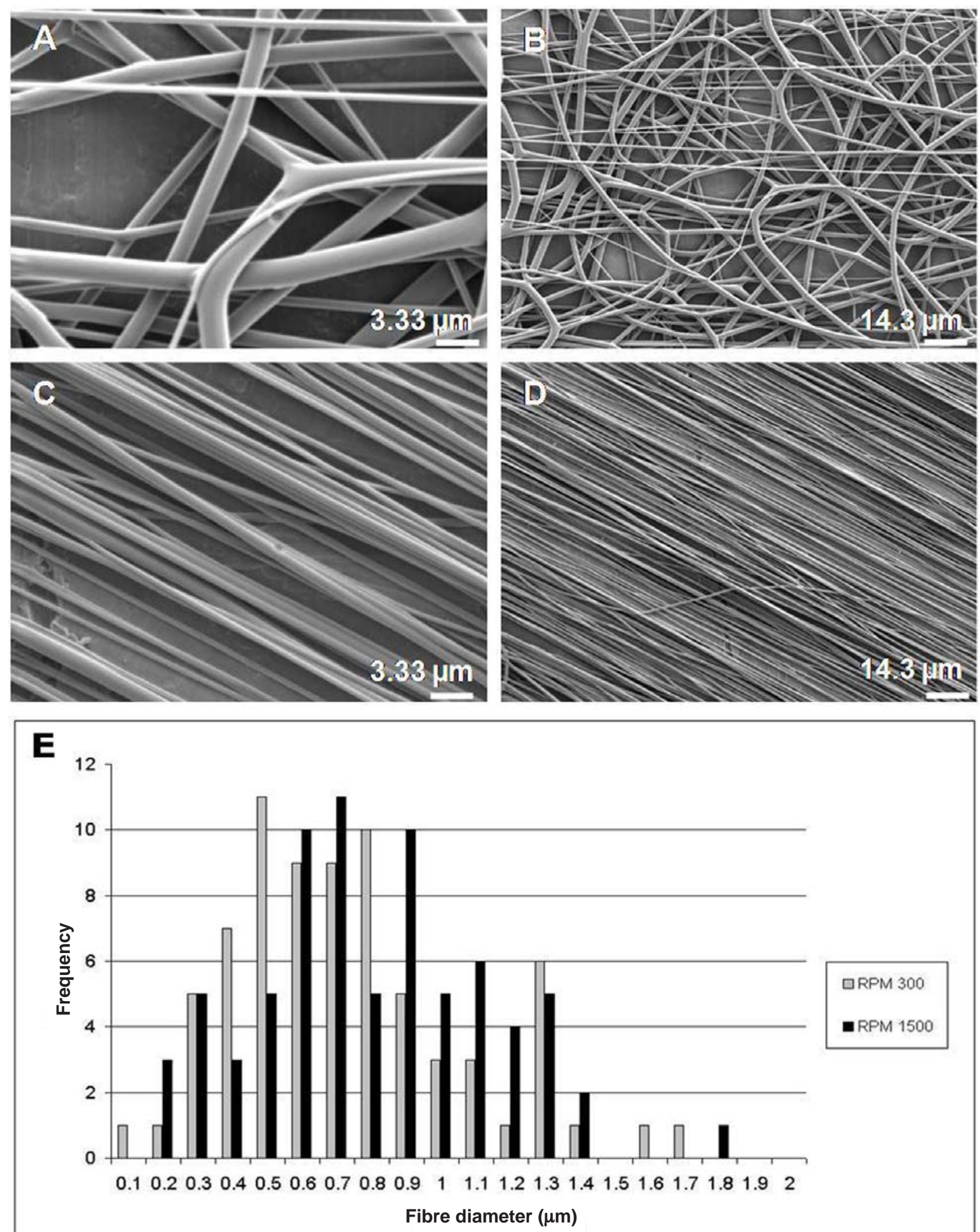

Figure 2: Scanning electron micrographs of RPM 300 (A and B) and RPM 1500 (C and D) electrospun PLGA fibres, (A) and (C) $3000 \times$ magnification, (B) and (D) $700 \times$ magnification; histogram (E) shows fibre diameter distribution in both fibre types. No significant difference was found between the fibre diameter of the different fibre types: $p>0.05$ as found by the students $t$-test ( $n=50$ per fibre type). 


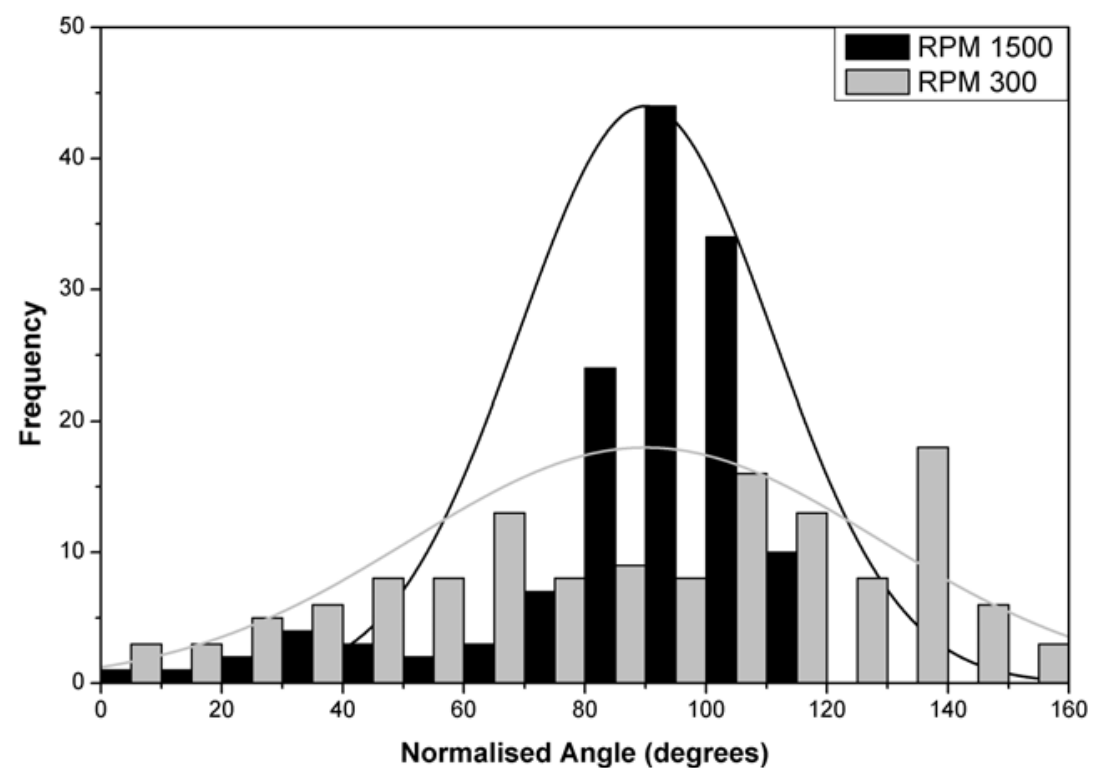

Figure 3: Histogram showing normalized (to 90 degrees) fibre angles in electrospun RPM1500 and RPM300 fibres. Black (RPM 1500) and grey (RPM 300) lines indicate the average for each cohort. The majority of fibres spun at RPM 1500 show alignment to 90-110 degrees. percentage elongation of 45\%, RPM 300 fibres tested in the same way had a percentage elongation of $50 \%$. Elongation of the fibres tested perpendicular to the directional axis as spun was much higher; RPM 1500 fibres elongated over $250 \%$, and RPM 300 fibres $175 \%$.

Micrographs A-D in Fig. 2 illustrate the observational differences between RPM 300 and RPM 1500; RPM 1500 have a more organized, aligned arrangement compared to RPM 300. These images suggest that RPM 1500 represents aligned electrospun fibres, where RPM 300 represents randomly oriented fibres. The histogram in Fig. 2 (E) illustrates the fibre diameter distribution as a function of frequency (number of fibres that lie in the fibre diameter group). There is a greater variety in fibre diameter in the RPM 300 fibres with the majority of fibre diameters within the $0.4-0.8 \mu \mathrm{m}$ range. In RPM 1500 fibres the diameter appears more controlled, with the majority of the fibre diameters within the $0.6 \mu \mathrm{m}-0.9 \mu \mathrm{m}$ range. RPM 1500 fibres appear, on average, $80 \mathrm{~nm}$ wider than RPM 300 fibres, but this is not significantly different $(p>0.05(0.52)$, $\mathrm{n}=50$ per fibre type).

Fibre alignment was quantified by measuring the mean fibre angle from 5 SEM images $(n=50$ per fibre type); these values were then normalized to 90 degrees and plotted in a histogram (Fig. 3) with the moving average line of best fit added to illustrate the distribution clearer. This histogram shows that RPM 300 have a broad distribution indicating lack of directional order, compared to RPM 1500 where the distribution is mainly between 80.5 to 110 degrees normalised to 90 degrees. Closer to 90 degrees indicates more alignment. Measuring the standard deviation of mean angles measured in the two fibre types also shows the variation in mean angle: a larger standard deviation would indicate more variation in angle measurements and less alignment, and vice versa. Standard deviation of each cohort of angle measurements was also calculated to show the amount of variation in each fibre type cohort (Table 1).

Results presented in Table 1 indicate that the RPM 1500 fibres show significantly more alignment $(p<0.05, \mathrm{n}=$
108 angles per fibre type) than RPM 300 fibres due to a significantly lower deviation value in RPM 1500 compared to RPM 300.

\section{Cellular alignment}

SEM analysis, shown in figure 4, allows visualization of both the cellular morphology and the orientation of the fibrous scaffolds. The cells appear to align along the directionality of the RPM 1500 fibres with an elongated morphology, and appear more polygonal, and less organised on the RPM 300 fibres. After 7 days culture, the cells on the RPM 300 fibres appear similar in morphology to those at the same time on RPM 1500 fibres, but looking at the overall directionality of the cells, it can be noted that on the RPM 300 there is a curvature in the direction of cell growth compared to the parallel growth of cells on RPM 1500.

Fluorescence staining for the f-actin component of the cytoskeleton shown in Fig. 5 illustrates how, after 30 minutes $\mathrm{C} 2 \mathrm{C} 12$ myoblast cells have adhered to all the surfaces. At this early time point the myoblasts on the RPM 1500 fibres have begun to organize their f-actin into parallel arrays of stress fibres, whereas on the RPM 300 fibres the f-actin is much less organised. On the coverslips, the actin is concentrated around the cell periphery indicating the active adhesion and spreading process. After 1 hour, the cells have formed a more defined cytoskeletal arrangement with stress fibres on the glass coverslips, and have more defined peripheral actin staining indicating the

Table 1: Standard deviation from mean of alignment in electrospun PLGA fibres $(\mathrm{n}=50)$. RPM1500 fibres showed significantly less deviation $(p<0.05)$ than RPM 300 fibres $(\mathrm{n}=108$ angles per fibre type).

\begin{tabular}{|c|c|c|}
\hline & RPM 300 & RPM 1500 \\
\hline Standard Deviation & 74.7 & 19.5 \\
\hline
\end{tabular}


RPM 1500

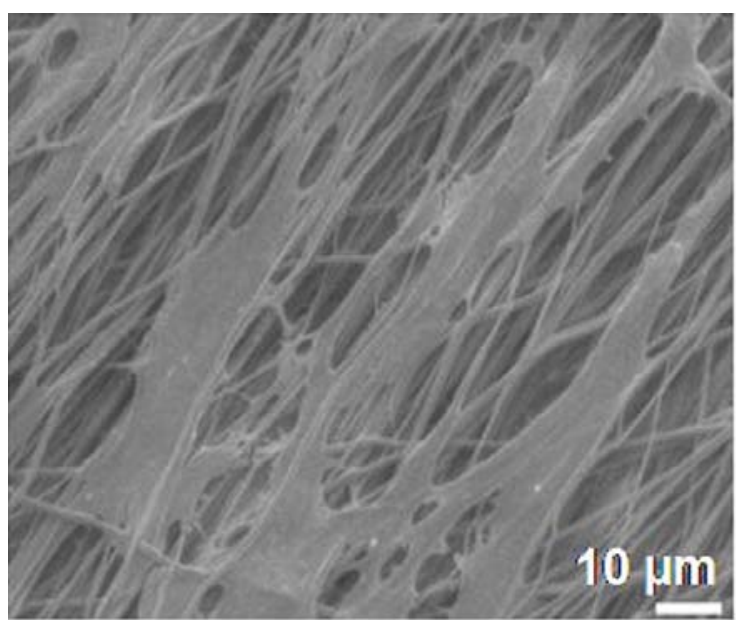

\section{4 hours}

3 days
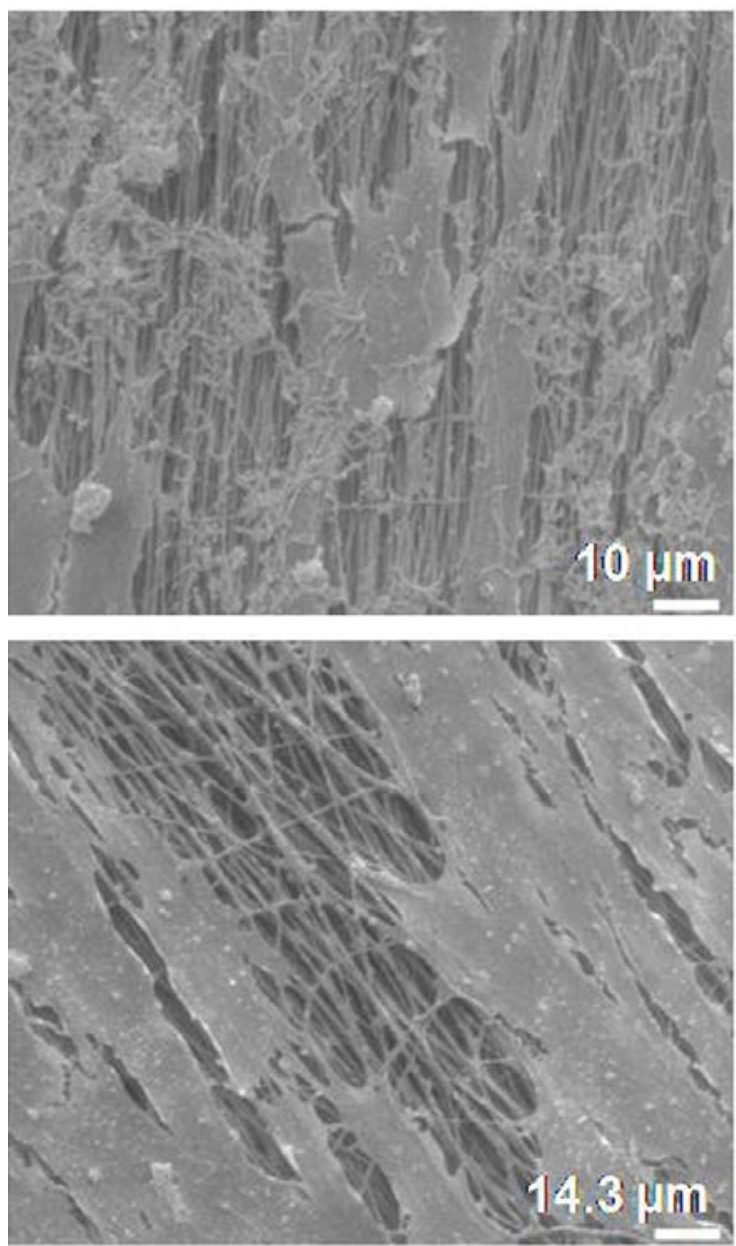

RPM 300
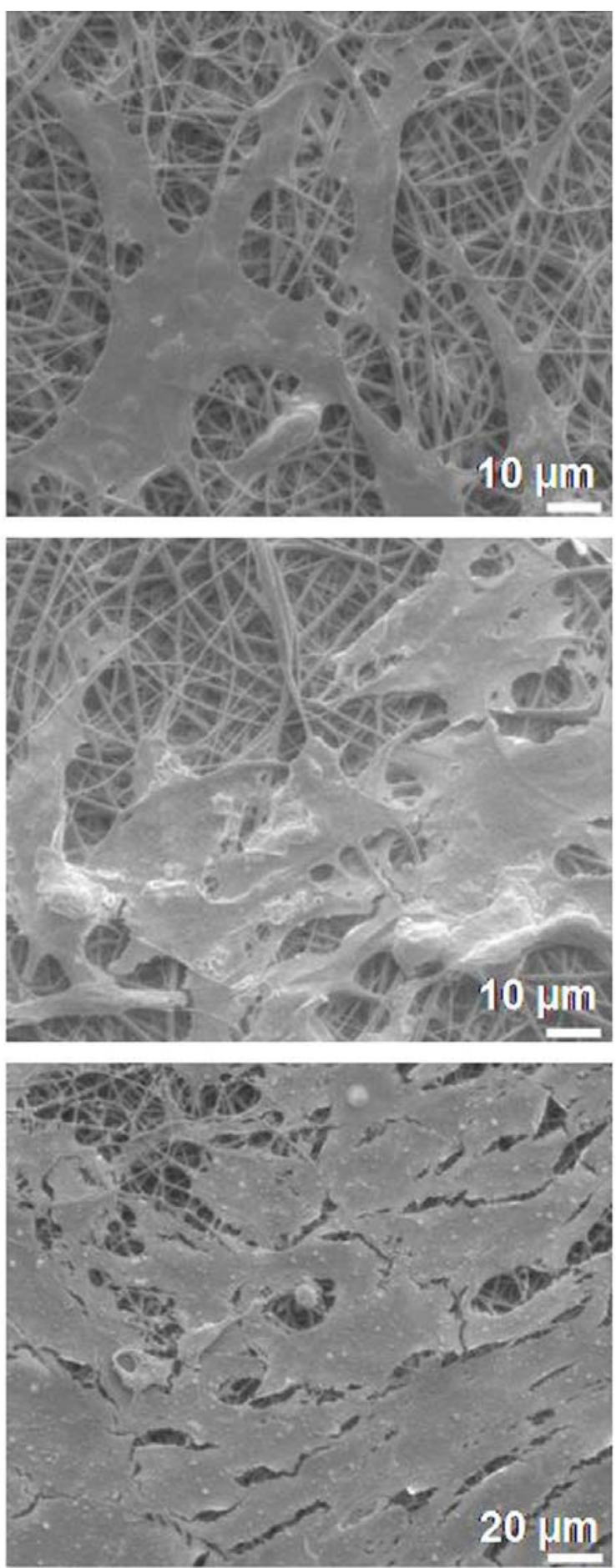

Figure 4: Scanning electron micrographs of myoblasts seeded on electrospun RPM 1500 and RPM 300 PLGA scaffolds. Myoblasts were fixed and viewed after 24 hours, 3 days, and 7 days in culture. Images show alignment of cells along the RPM 1500 fibres.

cells are still not fully spread on the glass surface. Cells upon the RPM 1500 show a degree of elongation with actin stress fibres becoming parallel compared to the RPM 300 fibres and the glass coverslips, where actin stress fibres create a polygonal cell morphology. By 24 hours the cells seeded on the glass coverslip control surface have a more polygonal morphology, compared to those seeded on the RPM 1500 electrospun fibres which have elongated in a bipolar manner. On the RPM 300 electrospun fibres, by 24 hours the cells are less polygonal than those on glass coverslips, but elongated to the same degree as those on RPM 1500 fibres after 5 hours.

Fig. 6 shows the quantification of elongation from the cells in figure 4 . To quantify the degree of elongation, the aspect ratio of the cells was measured ( $n=20$ per time point and sample type). The student's $t$-test was employed to determine any significant difference between the aspect ratios in each time point and sample type. After 30 minutes and 1 hour, elongation of cells on electrospun fibres is similar $(p>0.05)$, but is significantly different $(p<0.05)$ 


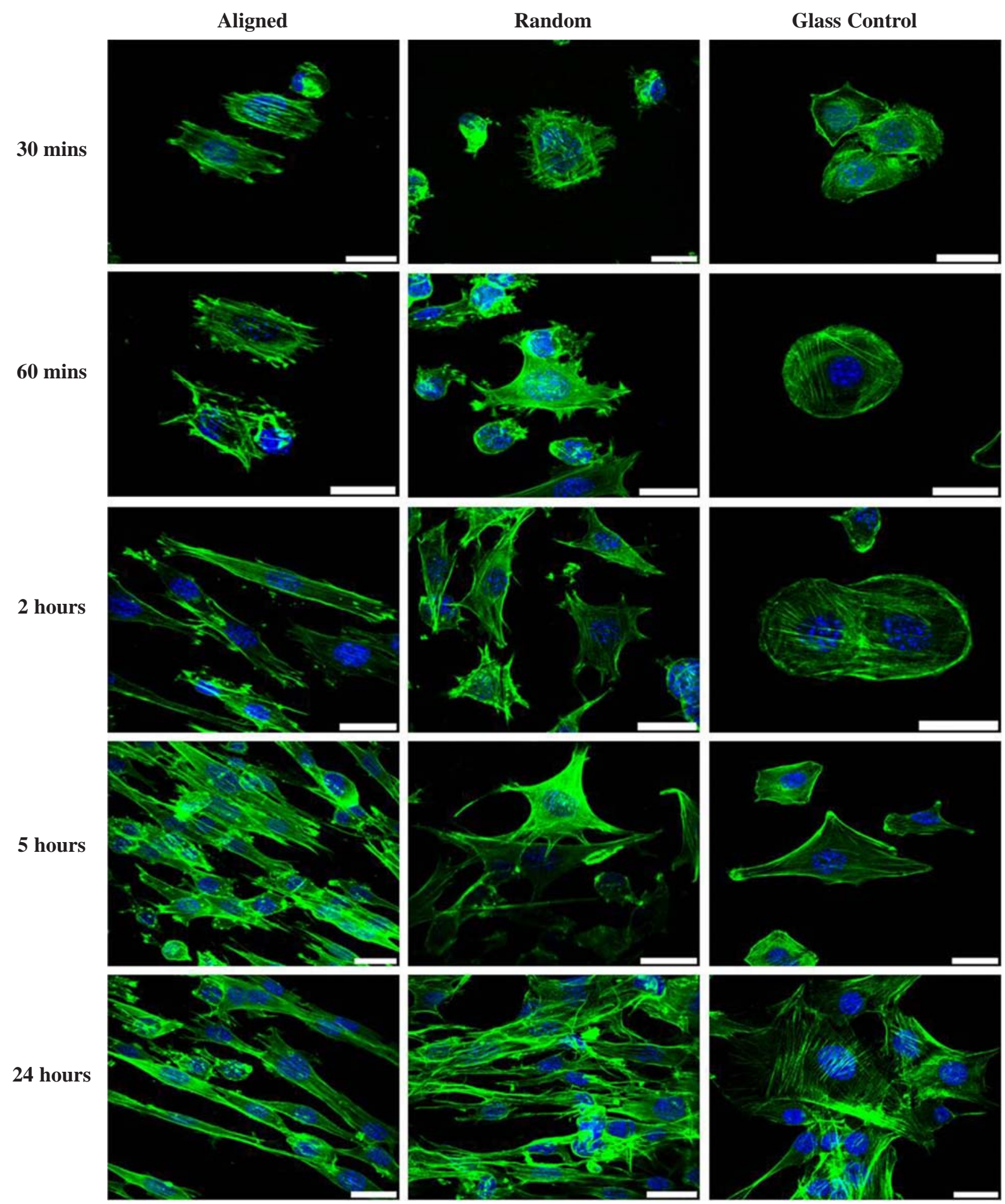

Figure 5: Confocal micrographs of myoblasts stained with FITC-phalloidin for the f-actin component of the cytoskeleton (green), and DAPI stained nuclei (blue). Cells were fixed and stained after 30 minutes, 60 minutes, 2 hours, 5 hours and 24 hours. There is evidence of cytoskeletal alignment along RPM 1500 fibres after just 30 minutes of culture. Scale bar indicates $25 \mu \mathrm{m}$.

from the glass control. From 2 hours all substrate types are significantly different from each other $(p<0.05)$. This result shows that all time points after 2 hours myoblasts are significantly more elongated on RPM 1500 fibres compared to both glass coverslips and RPM 300 fibres.

Figs. 4 and 5 illustrate the cellular morphology of the myoblast cells seeded on the electrospun scaffolds after relatively short time periods. Fig. 6 shows the cytoskeletal morphology of myoblasts, fixed and stained 14 days after seeding. These images show that the elongated morphology after 60 minutes seeding, on the RPM 1500 scaffold, is maintained throughout proliferation and differentiation. RPM 300 and the glass control show no parallel organization of the morphology of the cytoskeleton. 


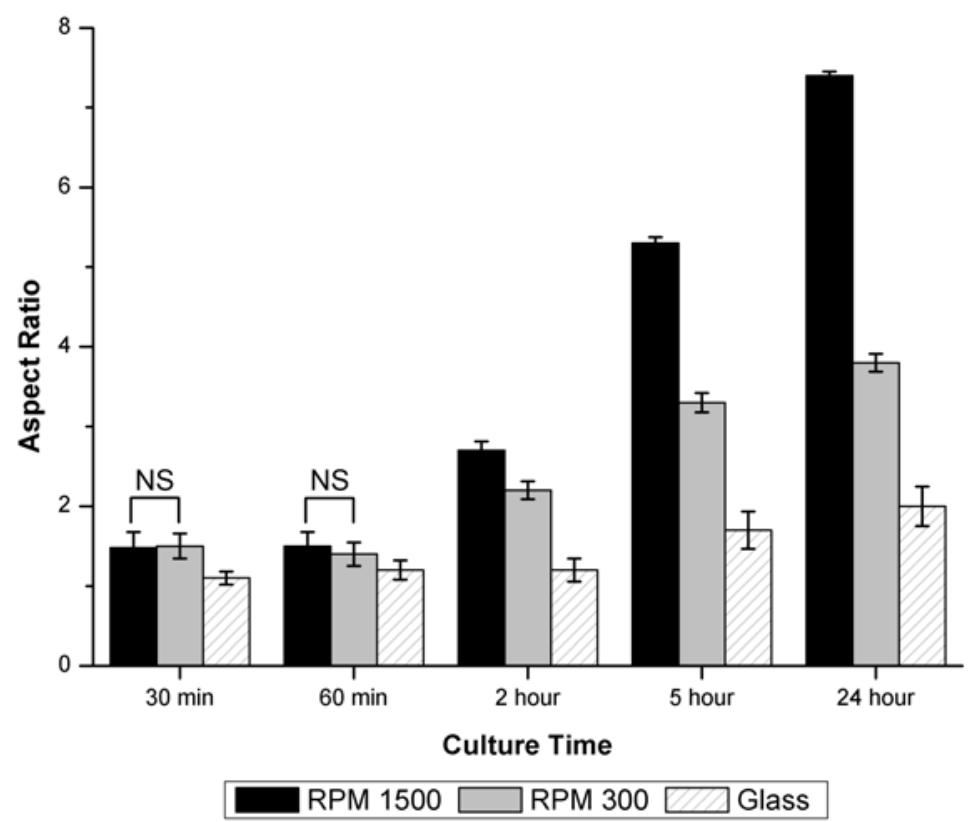

Figure 6: Quantification of elongation in myoblasts seeded on glass control and electrospun PLGA fibres. Values represent means \pm standard deviation, where $\mathrm{n}=20$. Brackets indicate not significantly different: $p>0.05$ as found by the student's $t$-test, all other data sets are significantly different from each other $(p<0.05)$. Cells are significantly more elongated on RPM 1500 fibres.
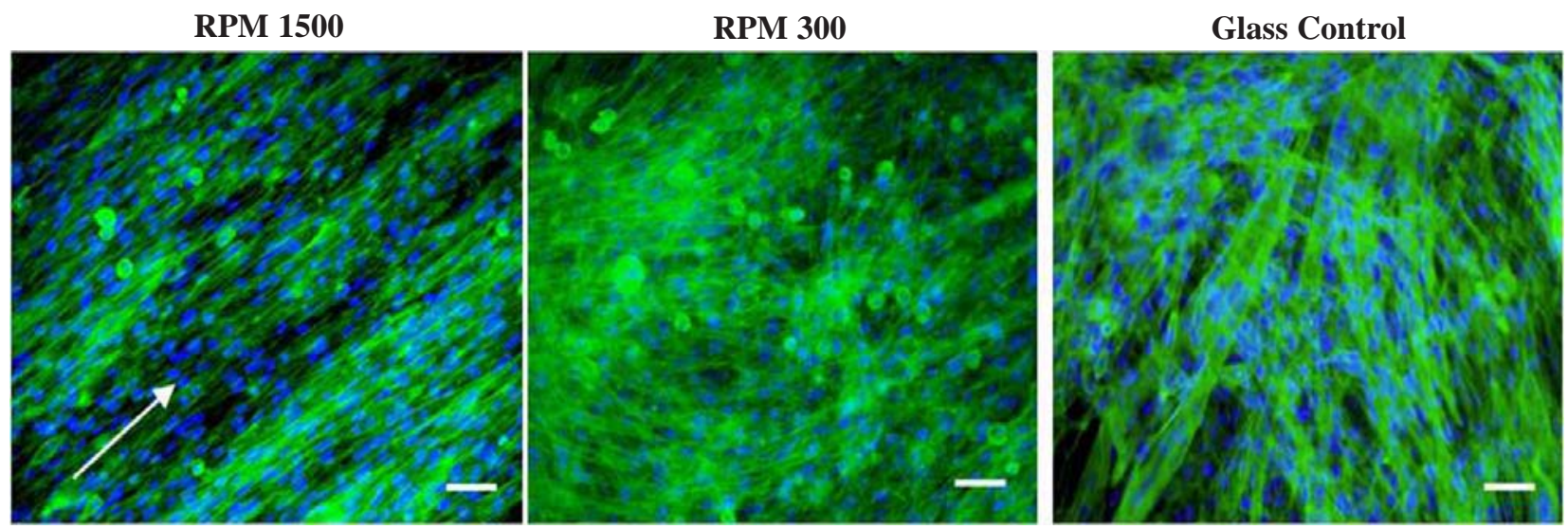

Figure 7: F-actin and nuclear staining, with FITC-phalloidin and DAPI respectively, of myoblast cells seeded on the electrospun scaffolds, fixed and stained after 14 days. Scale bar indicates $50 \mu \mathrm{m}$; arrow implies direction of cell alignment.

\section{Differentiation}

Differentiation of myoblasts into myofibres can be monitored by immunostaining of differentiation markers, proteins that are only expressed as the cell differentiates. Fast myosin heavy chain is a sarcomeric protein that is classically expressed after 7 days in cells in differentiation media.

Immunostaining of the differentiation marker fast myosin heavy chain (fast MyHC) is illustrated in Fig. 8. This result concurs with previous work (Engler et al., 2004) which suggests that myoblasts will differentiate more readily upon elastic or softer surfaces. This result shows that $\mathrm{C} 2 \mathrm{C} 12$ myoblasts will express normal differentiation proteins and begin to fuse into multinucleated myotubes, especially upon the electrospun PLGA fibres. The morphology of the cells shown in Fig. 8 show that the multinucleated myotubes are aligned in the same direction and are fused together to form long myofibre-like cells, compared to those on the glass where disordered fusion has taken place to create myotubes with a less fibre-like morphology.
Quantification of differentiation using low magnification $(10 \mathrm{x})$ ICC images of fast myosin heavy chain (fast MyHC) staining. To quantify the differentiation, the fusion index was calculated: number of fast $\mathrm{MyHC}$ positive multinucleated myotubes was divided by the total number of nuclei and converted into a percentage value, these values were then plotted as a bar chart as shown in Fig. 9. The graph in Fig.9 shows that after 14 days in culture, there is significantly more $(p<0.05)$ differentiation on RPM 1500 fibres compared to both glass and RPM 300 fibres.

\section{Contractility}

As discussed, myoblast and consequently myofibre elongation is thought to be essential for efficient contraction to take place. Immunostaining of a protein present in the sarcomere allows visualization of the capability to contract.

Low magnification images of myoblast cells stained for sarcomeric myosin (red) shown in Fig. 10 not only illustrate the distinct, and expected cell elongation along 
RPM 1500
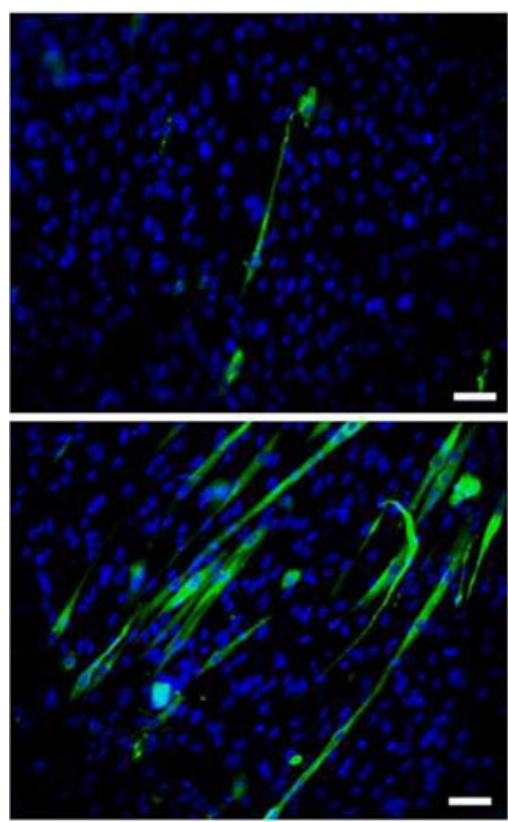

Day 7

Day 14

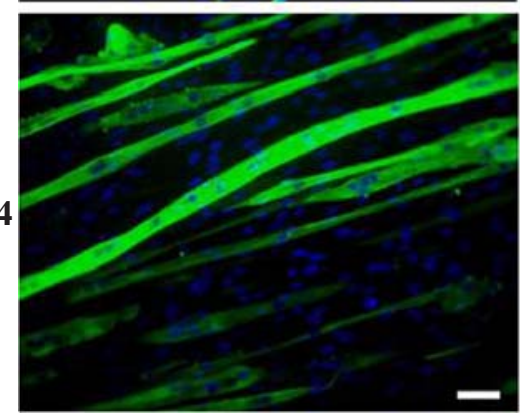

RPM 300
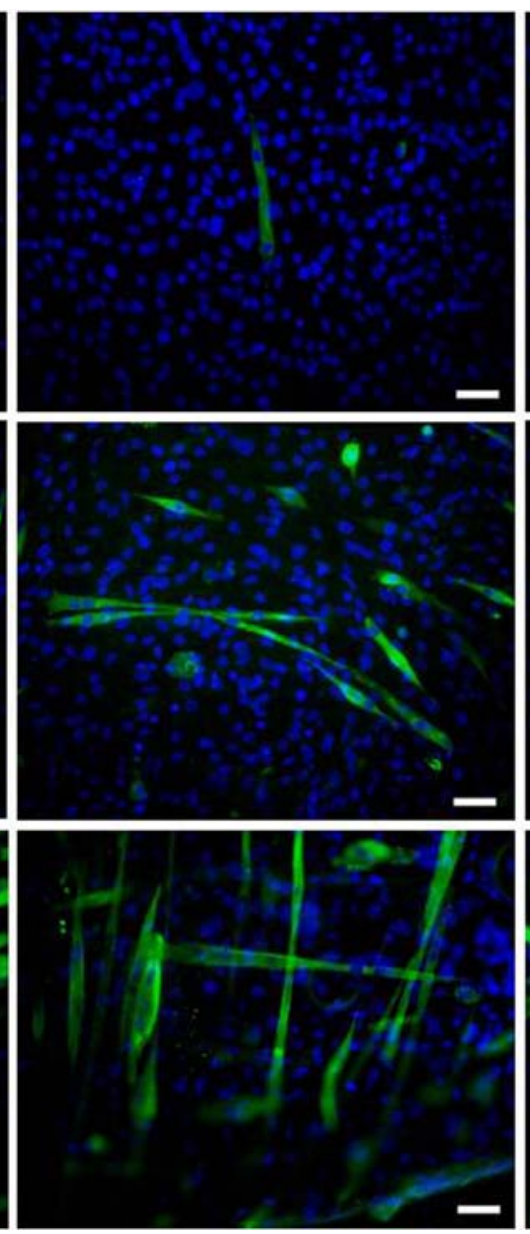

Glass Control
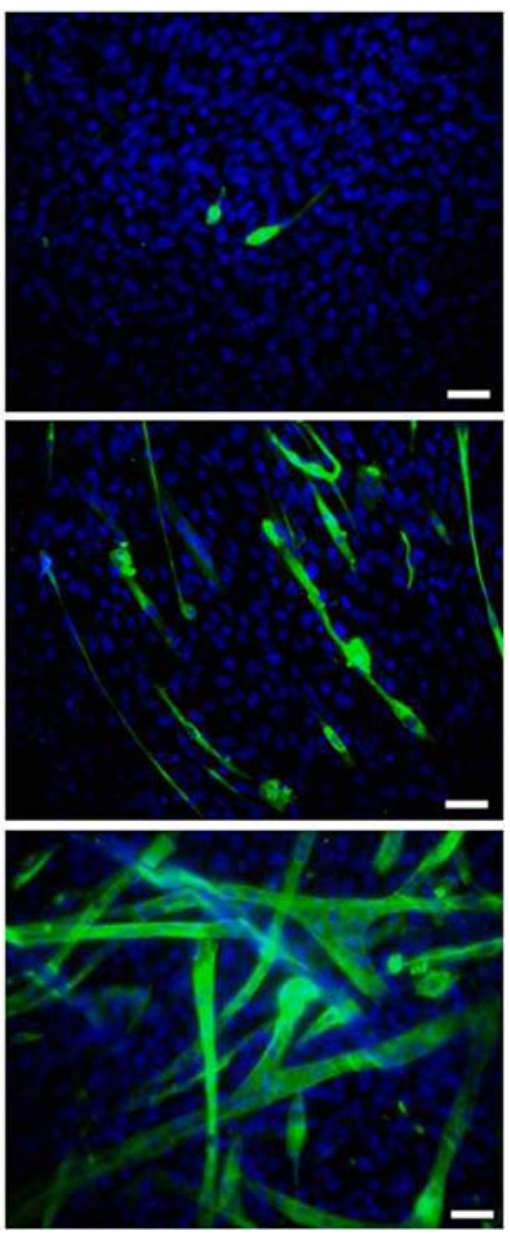

Figure 8: Fluorescence micrographs of myoblast cells stained for fast myosin heavy chain (green) and nuclear staining (blue). Cells were fixed and stained after 3 days, 7 days, and 14 days in differentiation media. Scale bar indicates 50 $\mu \mathrm{m}$ (magnification $20 \mathrm{x}$ ).

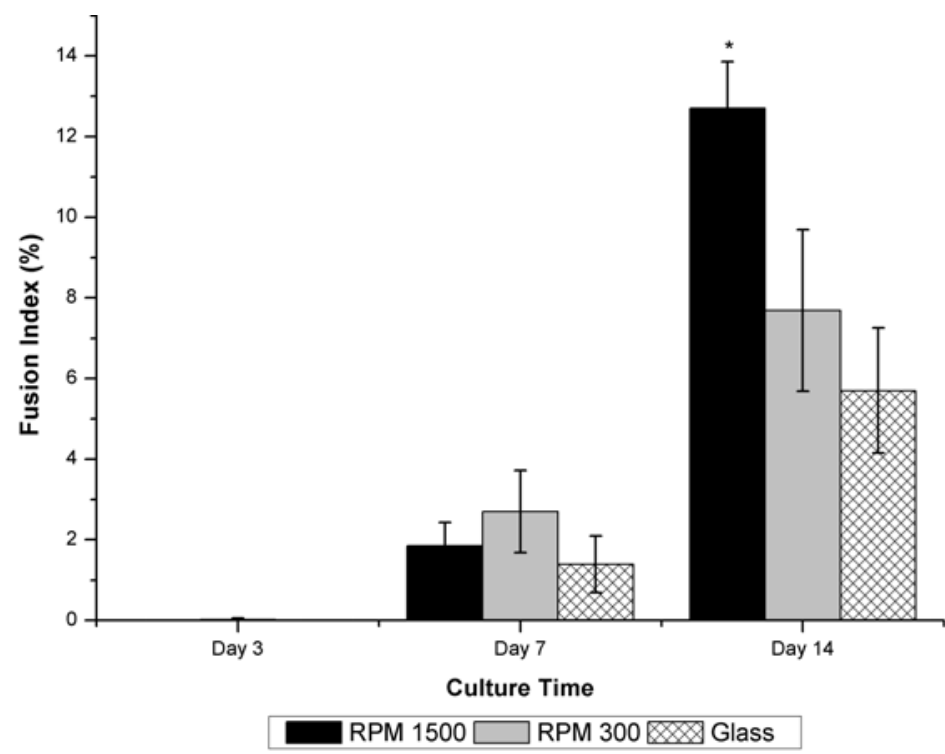

Figure 9: Graph presenting percentage fast myosin heavy chain positive multinucleated myotubes, counted using ImageJ software. Bars indicate standard deviation, star indicates significant difference $(p<0.05, \mathrm{n}=5$ per substrate type and time point) as shown by students' $t$-test. There is a significantly higher number of differentiated myotubes on the RPM 1500 fibres. one axis as shown in figures 4,5 , and 6 , but also shows the expression of sarcomeric myosin. This expression, as shown by red staining in Fig. 10 is more abundant on RPM 1500 fibres compared to RPM 300 and the glass control.

Confocal images shown in Fig. 11 illustrate the indication of correct sarcomeric protein organization.
These images show sarcomeric myosin in red, it is these red striations that indicate correct organization, as they represent the myosin in the A band of the sarcomere. From these images it can be concluded that the myotubes formed on the electrospun fibres are able to organize the correct sarcomeric protein array. 

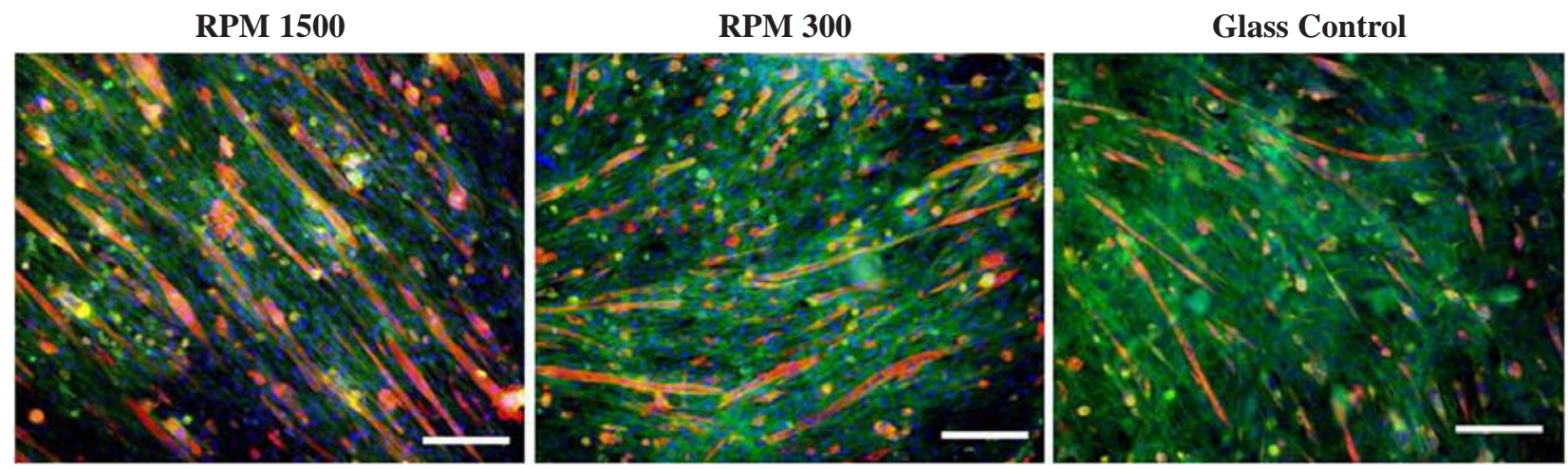

Figure 10: Myoblast cells fixed and stained for sarcomeric myosin (red), f-actin (green), and nuclei (blue) after 14 days culture in differentiation media. Original magnification is $10 \mathrm{x}$; scale bar indicates $200 \mu \mathrm{m}$.
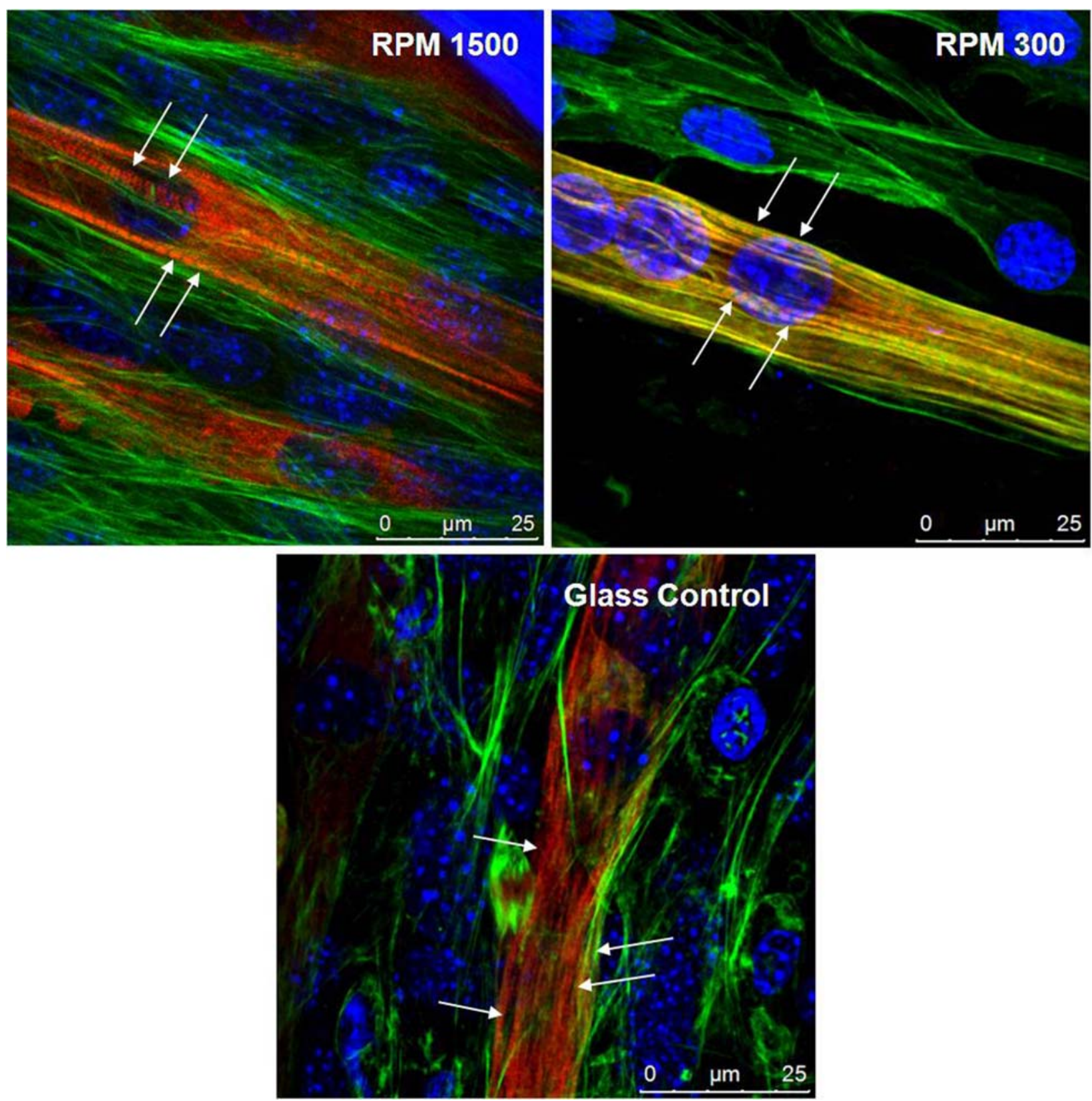

Figure 11: Confocal micrographs of myoblasts seeded on electrospun scaffolds and glass control stained for sarcomeric myosin (red), f-actin (green), and nuclei (blue) after 14 days in differentiation media. Arrows point to individual bands of sarcomeric myosin; scale bar shows $25 \mu \mathrm{m}$. 


\section{Discussion}

Regeneration of a fully functioning skeletal muscle patch for grafting or as a true-to-life research tool for diseases affecting the tissue is a challenge for bioengineers. Creating and maintaining cellular organization and architecture being the primary difficulty. Synthetic materials, such as PLGA, may create a biodegradable scaffold with the topography to introduce contact guidance to the cells in order for them to grow parallel to each other in one direction (Bian and Bursac, 2009; Choi et al., 2008; Lam et al., 2009). Contact guidance is the reaction of cells to topographical features (Dalby et al., 2003). Berendse et al. (2003) and Peckham (2008) report that parallel f-actin cytoskeletal arrangement is important for successful sarcomeric formation (Berendse et al., 2003; Peckham, 2008). Due to the inherent alignment within the electrospun PLGA scaffold described in this paper and the profound effect on cellular alignment and cytoskeletal arrangement it could become the basis for eventual 3D tissue culture incorporating different cell types for vascularisation. This type of electrospun scaffold does not allow for cellular infiltration thus would be used as a template to create and maintain the alignment of the myoblast cells. Used in conjunction with other materials e.g. a gel it could be used to create a 3D tissue construct. Levenberg et al. (2005) created a PLLA and PLGA porous foam and seeded it with a tri-culture of cells to encourage vascularisation to occur simultaneously with myoblast differentiation (Levenberg et al., 2005). Lam et al. (2009) used a PDMS scaffold as a template for aligned myotube growth then transferred the cells onto a fibrin gel for 3D cell infiltration into the gel, maintaining the alignment (Lam et al., 2009).

In the current study it is shown that highly aligned electrospun PLGA fibres (RPM 1500) can be reproducibly made with diameters ranging from $100 \mathrm{~nm}$ to $1.4 \mu \mathrm{m}$. This alignment can be quantified by measuring the standard deviation from the mean; randomly oriented fibres (RPM 300 in this study) would be expected to have a large standard deviation, compared to aligned fibres (RPM 1500) that would yield a smaller standard deviation. Our results concur with this theory; RPM 300 fibres have a larger standard deviation than RPM 1500 fibres. It is also shown that this highly aligned scaffold can provide the topographical cues for the alignment of $\mathrm{C} 2 \mathrm{C} 12$ murine myoblasts. Using both SEM and fluorescence imaging it is possible to illustrate that myoblasts elongate and align along the parallel axis of the electrospun PLGA fibres. Quantification of elongation from the fluorescence images statistically shows that the cellular morphology on aligned fibres (RPM 1500) is more elongated; looking at this in conjunction with the SEM images the cellular morphology responds to the alignment in the scaffold, and is maintained through proliferation over 14 days. Along with controlling cellular organisation with aligned sub-micron fibres, the PLGA scaffolds also encourage myoblast differentiation. Quantification of differentiation, measured by number of multinucleated ( $>2$ nuclei) myotubes expressing the differentiation marker fast myosin heavy chain as shown in Fig. 9 indicates around 13\% multinucleated myotubes present on the RPM 1500 fibres which is significantly higher $(p<0.05)$ than those on RPM 300 fibres or the glass control. This figure is relative to other studies where no biologic components were added to the cellular substrate (Stern et al., 2009). This differentiation, along with the immunostaining of sarcomeric myosin (see Figs. 10 and 11), show that myotube alignment introduced by the electrospun fibres leads to preferential expression of differentiation markers.

This could be due to the myoblasts being organised to encourage fusion and consequent differentiation. Results shown in Figs. 10 and 11 illustrate that the sarcomeric contractile machinery is arranged correctly forming the typical banding pattern characteristic of skeletal muscle fibres. This banding is clearer and more defined in myotubes seeded on the electrospun scaffolds. Due to this stereotypical highly organized arrangement of the contractile proteins the cells have the ability to contract when given the appropriate stimulus. Levi-Mishali et al. (2009) showed that PLGA scaffolds with at least 50:50 lactide:glycolide ratio are elastic enough to allow myotube formation without detatchment, this study uses PL85GA15 which falls into the criteria stated by Levi-Mishali et al. stating what elasticity is optimum for myoblast differentiation (Levi-Mishali et al., 2009).

Previous studies have shown that aligned fibres can influence the orientation of the cell type seeded upon them (Bashur et al., 2006; Bian and Bursac, 2009; Choi et al., 2008; Courtney et al., 2006; Huang et al., 2006; Lehnert et al., 2004; Riboldi et al., 2005; Wang et al., 2009). Other studies have incorporated natural biologic materials e.g. collagen, to improve biocompatibility (Choi et al., 2008; Lam et al., 2009). This study illustrates a totally synthetic scaffold with no surface modifications or feeder layer/coculture can support the attachment, proliferation and differentiation of $\mathrm{C} 2 \mathrm{C} 12$ myoblasts. Future work includes an in vitro degradation study comparing random and aligned electrospun fibres with spin coated PLGA, looking at mass loss, surface morphology changes, $\mathrm{pH}$ changes, and molecular weight modulations.

\section{Acknowledgements}

The BBSRC is gratefully acknowledged for funding the research studentship (KJA) and Confocal microscope (BB/ FO11547/1). Thanks to Dr. Steve Eichhorn and The Royal Society for use and funding of the electrospinning equipment.

\section{References}

Bashur CA, Dahlgren LA, Goldstein AS (2006) Effect of fiber diameter and orientation on fibroblast morphology and proliferation on electrospun poly(D,L-lactic-coglycolic acid) meshes. Biomaterials 27: 5681-5688.

Berendse M, Grounds MD, Lloyd CM (2003) Myoblast structure affects subsequent skeletal myotube morphology and sarcomere assembly. Exp Cell Res 291: 435-450.

Bian W, Bursac N (2009) Engineered skeletal muscle tissue networks with controllable architecture. Biomaterials 30: 1401-1412. 
Blackwood KA, McKean R, Canton I, Freeman CO, Franklin KL, Cole D, Brook I, Farthing P, Rimmer S, Haycock JW (2008) Development of biodegradable electrospun scaffolds for dermal replacement. Biomaterials 29: 3091-3104.

Bray M-A, Sheehy SP, Parker KK (2008) Sarcomere alignment is regulated by myocyte shape. Cell Motil Cytoskel 65: 641-651.

Choi J S, Lee S J, Christ G J, Atala A, Yoo J J (2008) The influence of electrospun aligned poly(caprolactone)/ collagen nanofiber meshes on the formation of self-aligned skeletal muscle myotubes. Biomaterials 29: 2899-2906.

Cooper S T, Maxwell A L, Kizana E, Ghoddusi M, Hardeman E C, Alexander I E, Allen D G, North K N (2004) C2C12 co-culture on a fibroblast substratum enables sustained survival of contractile, highly differentiated myotubes with peripheral nuclei and adult fast myosin expression. Cell Motil Cytoskel 58: 200-211.

Courtney T, Sacks M S, Stankus J, Guan J, Wagner W $R$ (2006) Design and analysis of tissue engineering scaffolds that mimic soft tissue mechanical anisotropy. Biomaterials 27: 3631-3638.

Dalby M J, Childs S, Riechle MO, Johnstone HJH, Affrossman S, Curtis ASG (2003) Fibroblast reaction to island topography: changes in cytoskeleton and morphology with time. Biomaterials 24: 927-935.

Engler J, Griffin MA, Sen S, Bonnemann CG, Sweeny HL, Discher DE (2004) Myotubes differentiate optimally on substrates with tissue-like stiffness: pathological implications for soft of stiff microenvironments. J Cell Biol 166: $877-887$.

Huang NF, Patel S, Thakar RG, Wu J, Hsiao BS, Chu B, Lee RJ, Lee S (2006) Myotube assembly on nanofibrous and micropatterned polymers. Nano Letters 6: 537-542.

Huang Y-C, Dennis RG, Larkin L, Baar K (2005) Rapid formation of functional muscle in vitro using fibrin gels. J App Phys 98: 706-713.

Kaetsu I, Yoshida M, Asano M, Yamanaka H, Imai K, Yuasa H, Mashimo T, Suzuki K, Katakai R, Oya M (1987) Biodegradable implant composites for local therapy. J Control Release 6: 249-263.

Kim K, Yu M, Zong X, Chiu J, Fang D, Seo Y-S, Hsiao BS, Chu B, Hadjiargyrou M (2003) Control of degradation rate and hydrophillicity in electrospun non-woven poly(D,L-lactide) nanofiber scaffolds for biomedical applications. Biomaterials 24: 4977-4985.

Lam MT, Huang Y-C, Birla RK, Takayama S (2009) Microfeature guided skeletal muscle tissue engineering for highly organised 3-dimensional free-standing constructs. Biomaterials 30: 1150-1155.

Lehnert D, Wehrle-Haller B, David C, Weiland U, Ballestrem C, Imhof BA, Bastmeyer M (2004) Cell behaviour on micropatterned substrata: limits of extracellular matrix geometry for spreading and adhesion. J Cell Sci 117: 41-52.

Levenberg S, Rouwkema J, Macdonald M, Garfein ES, Kohane DS, Darland DC, Marini R, Van Blitterswijk CA, Mulligan RC, D’Amore PA (2005) Engineering vascularised skeletal muscle tissue. Nat Biotechnol 23: 879-884.

Levi-Mishali M, Zoldan J, Levenberg S (2009) Effect of scaffold stiffness on myoblast differentiation. Tissue Eng 15: 935-944.

Peckham M (2008) Engineering a multinucleated myotube, the role of the actin cytoskeleton. J Microsc 231: 486-493.

Ren K, Crouzier T, Roy C, Picart C (2008) Polyelectrolyte multilayer films of controlled stiffness modulate myoblast differentiation. Adv Funct Mater 18:1378-1389.

Riboldi S A, Sampaolesi M, Neuenschwander P, Cossu G, Mantero S (2005) Electrospun degradable polyesterurethane membranes: potential scaffolds for skeletal muscle tissue engineering. Biomaterials 26: 46064615.

Schiaffino S, Partridge T (eds) (2008) Skeletal Muscle Repair and Regeneration, Advances in Muscle Research, Vol 3. Springer Netherlands, Dordrecht.

Singh L, Kumar V, Ratner B D (2004) Generation of porous microcellular $85 / 15$ poly(DL-lactide-co-glycolide) foams for biomedical applications. Biomaterials 25: 26112617.

Stern M, Myers R L, Hammam N, Stern KA, Eberli D, Kritchevsky SB, Soker S, Dyke M V (2009) The influence of extracellular matrix derived from skeletal muscle tissue on the proliferation and differentiation of myogenic progenitor cells ex vivo. Biomaterials 30: 2393-2399.

Subbiah T, Bhat G S, Tock R W, Parameswaran S, Ramkumar S S (2004) Electrospinning of nanofibres. J Appl Polym Sci 96: 557-569.

Wang HB, Mullins ME, Cregg JM, Hurtado A, Oudega M, Trombley MT, Gilbert RJ (2009) Creation of highly aligned electrospun poly-L-lactide acid fibres for nerve regeneration applications. J Nerual Eng 6: 016001 (15pp).

Webb AR, Yang J, Ameer GA (2004) Biodegradable polyester elastomers in tissue engineering. Exp Opin Biol Ther 4: 801-812.

Zong X, Bien H, Chung C-Y, Yin L, Fang D, Hsiao BS, Chu B, Entcheva E (2005) Electrospun fine-textured scaffolds for heart tissue constructs. Biomaterials 26: 53305338.

Zong X, Ran S, Kim K-S, Fang D, Hsiao BS, Chu B (2003) Structure and morphology changes during in vitro degradation of electrospun poly(glycolide-co-lactide) nanofibre membrane. Biomacromolecules 4: 416-423. 